\title{
ASS reduziert das Kolon-Ca.-Risiko
}

\section{Bei Patienten mit hereditär bedingt besonders hohem Risiko für kolorek- tale und andere Karzinome reduziert die langfristige ASS-Prophylaxe das Karzinomrisiko.}

- Durch prophylaktische Aspirin-Einnahme scheint sich die Inzidenz kolorektaler Karzinome vermindern zu lassen. Aufgrund des notwendigen langen Nachsorgezeitraums konnte dieser Effekt in randomisiert kontrollierten Studien bisher lediglich für kolorektale Adenome bestätigt werden, nicht jedoch für Karzinome. Burn et al. untersuchten nun die Langzeitergebnisse der bereits 2008 im New England Journal of Medicine publizierten CAPP2-Patientengruppe.

In die CAPP2-Studie wurden initial 1071 Patienten mit hereditären nicht polypösen Kolonkarzinomen (HNPCC, Lynch Syndrom) prospektiv für eine zweijährige Therapie randomisiert. Die Behandlungsarme verglichen dabei unter anderem $600 \mathrm{mg}$ Acetylsalicylsäure täglich vs. Placebo. Nun wurden die Langzeitdaten von 861 Patienten nach

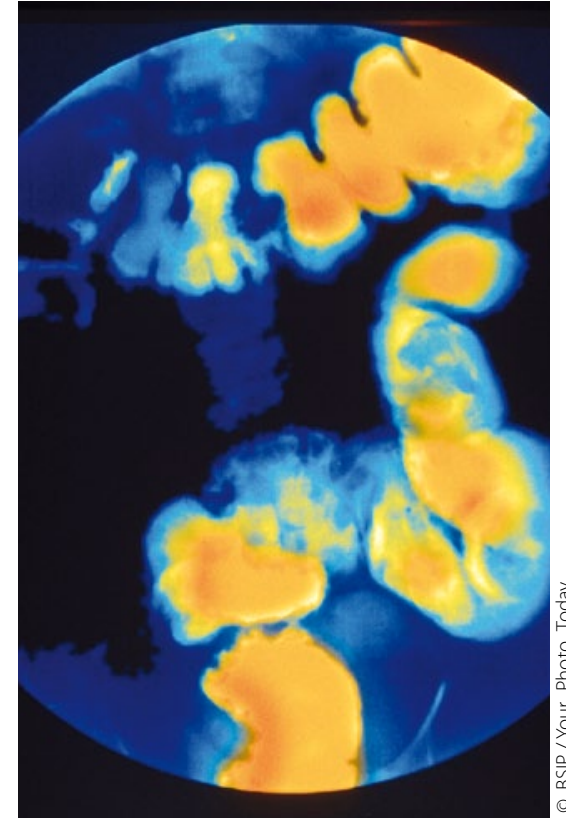

Kolorektales Karzinom: Bei hohem Risiko wirkt ASS prophylaktisch.

einer medianen Nachsorgezeit von fast fünf Jahren im Hinblick auf die Entwicklung eines kolorektalen Karzinoms analysiert.
Das Risiko für die Entwicklung eines kolorektales Karzinoms betrug in der Aspiringruppe 4,2\% (18 von 427) und in der Placebogruppe 6,9\% (30 von 434). In der intention to treat Analyse bis zum Auftreten des ersten Karzinoms erwies sich dieser Unterschied als nicht signifikant $(p=0,12)$. Wurden jedoch die fünf beobachteten Doppelkarzinome in der Auswertung mittels Poisson Regressionsanalyse berücksichtigt, so zeigte sich ein geringeres Karzinomrisiko für die ASS-Gruppe $(p=0,05)$. Die per protocol Analyse zeigte, dass der Effekt für diejenigen Patienten am stärksten war, die die geplante Therapiedauer von zwei Jahren einhielten ( $p=0,02$ für den Zeitraum bis zum ersten kolorektalen Karzinom und $\mathrm{p}=0,008$ in der Poisson Regressionsanalyse). Es bestand kein signifikanter Unterschied zwischen ASS- und Placebogruppe bezüglich Nebenwirkungen oder Patientencompliance.

- J. Burn et al.

Long-term effect of aspirin on cancer risk in carriers of hereditary colorectal cancer: an analysis from the CAPP2 randomised controlled trial. Lancet 378 (2011) 2081-2087

\section{Kommentar}

Patienten mit HNPCC haben ein 80-prozentiges Lebenszeitrisiko für die Entwicklung eines kolorektalen oder anderen HNPCCassoziierten Karzinoms. In bisherigen Studien erscheint die prophylaktische ASS-Behandlung vielversprechend. Jedoch stützen sich diese Aussagen auf retrospektive Daten oder auf die Adenombildung als primären Endpunkt, nicht aber auf die Entwicklung kolorektaler Karzinome.

Die Ergebnisse der Studie von Burn et al. bestätigen nun den protektiven Effekt einer ASS-Langzeitbehandlung bei Patienten mit HNPCC. Allerdings wird das Signifikanzniveau in der einfachen intention to treat Analyse nicht erreicht $(p=0,12)$. Die Poisson Regressionsanalyse, welche fünf Patienten mit Doppelkarzinom gesondert berücksichtigt, ergab einen grenzwertig signifikanten Effekt für die ASS-Behandlung $(p=0,05)$. Schließlich zeigte sich der größte Nutzen für Patienten, die mindestens zwei Jahre regelmäßig ASS einnahmen ( $p=0,02$ bzw. $p=0,008)$. Erwähnenswert ist, dass insgesamt 40 Patienten ein nicht kolorektales Lynch-Karzinom entwickelten. Fasst man alle Lynch-Karzinome zusammen (neben Kolon und Rektum auch Endometrium, Ovar, Pankreas, Dünndarm, Gallenblase, Ureter, Magen, Niere und Zerebrum), so ließ sich ebenfalls ein protektiver Effekt für die Aspirin-Therapie erkennen. Bei der Analyse aller Lynch-Karzinome wurde eine Dosisabhängigkeit des Effekts festgestellt.
Die derzeitige Datenlage deutet darauf hin, dass eine langfristige prophylaktische Aspirin-Einnahme das Auftreten kolorektaler Karzinome vermindert. Davon profitieren insbesondere Risikogruppen wie HNPCCPatienten. In der hier vorgestellten Studie wurde mit $600 \mathrm{mg}$ Aspirin eine relativ hohe tägliche Dosis untersucht. Der protektive ASS-Effekt zeigte sich je nach statistischer Auswertung und Dauer der Einnahme sowie Nachsorge als grenzwertig signifikant. Daher ist nun von der gleichen Forschergruppe die CAPP3-Studie in Planung, welche sich mit der optimalen Dosis und Behandlungsdauer befasst.

U. NitSCHE, F. G. BADER, KLINIKUM RECHTS DER ISAR, MÜNCHEN - 